ARTíCULO

\title{
Biología reproductiva de Mulloidichthys dentatus (Perciformes: Mullidae) en el Pacífico tropical mexicano
}

Reproductive biology of Mulloidichthys dentatus (Perciformes: Mullidae) in the Mexican tropical Pacific

\section{Gabriela Lucano-Ramírez', Maricruz Robles-Raveleroํ, Salvador Ruiz-Ramírez ${ }^{1 *}$, Gaspar González-Sansón ${ }^{1,2}$, Consuelo Aguilar-Betancourt ${ }^{1,2}$ y Alejandro Perez-Toledo ${ }^{1}$}

\author{
${ }^{1}$ Departamento de Estudios para el Desarrollo Sustentable de Zonas Costeras, Universidad de Guadalajara, V. Gómez Farías 82, San \\ Patricio-Melaque, Cihuatlán, Jalisco, C.P. 48980, México \\ ${ }^{2}$ Canadian Rivers Institute, 100 Tucker Park Rd, Saint John, NB E2L 4A6, Canadá \\ *Autor corresponsal: salruizram@hotmail.com
}

\begin{abstract}
Although Mulloidichthys dentatus is a commercially important species in this region of the Mexican Pacific, most aspects of its biological life cycle, including reproduction, are not known. The main goal of this study was the description of several reproductive traits of $M$. dentatus in the southern coast of Jalisco, Mexico. Monthly sampling was carried on from January 1998 to December 2008. Fish were caught using a set of gill nets with different mesh sizes. A total of 1,590 individual were sampled and total length (TL), total weight, sex and maturation stage were determined for each fish. Gonads were extracted and preserved for histological analyses. Minimum, mean and maximum TL of fish sampled were 16.5, 30.9 and $40.0 \mathrm{~cm}$, respectively. A total of 899 females and 691 males were collected with the overall sex ratio equal to 1.3:1. Maximum values of gonadosomatic index (GSI) and mean oocyte diameter, as well as the percentages of gonads in advanced reproduction stages suggest that $M$. dentatus has three main reproductive periods in March, May-July and November. Significant correlation between both sexes was found for GSI and condition factor. Seven oocyte development phases were found in ripe ovaries, which suggest asynchronic oocyte development. Cell arrangement found in testis was of lobular type with an externally well-defined sperm duct. Maturation length was $25.4 \mathrm{~cm}$ for females and $24.5 \mathrm{~cm}$ for males. As these values are less than the mean lengths observed in catches ( 31.9 and $29.7 \mathrm{~cm} \mathrm{TL}$ ), it can be assumed that more than $50 \%$ of caught individuals have had a reproductive event (99\% female and male).
\end{abstract}

Key words: Goatfish, reproductive period, maturity size, sex ratio, gonadosomatic index

Resumen.- Aunque Mulloidichthys dentatus se captura de manera comercial en esta región del Pacífico mexicano, no se conoce gran parte de su biología básica, incluida la reproducción. Este estudio describe aspectos reproductivos de $M$. dentatus en la costa sur de Jalisco, México. El trabajo se realizó entre enero de 1998 a diciembre de 2008 con muestreos mensuales. Los organismos fueron capturados con redes de enmalle de diferente luz de malla. Se obtuvieron 1.590 organismos, a los cuales se les registró la longitud total, peso total, se les extrajeron las gónadas, se determinó el sexo, se evaluó el estadio de madurez y se procesaron histológicamente las gónadas. Las longitudes mínima, promedio y máxima fueron 16,5, 30,9 y 40,0 cm, respectivamente. Del total de organismos, 899 fueron hembras y 691 machos. La proporción sexual fue dominada por las hembras (1,3:1 H:M). Los valores máximos del índice gonadosomático (IGS), del diámetro promedio de los ovocitos, así como los porcentajes de organismos con gónada en estadio capaz de reproducirse, sugieren que $M$. dentatus presenta tres periodos reproductivos: en marzo, de mayo a julio y en noviembre. Se encontró correlación temporal entre sexos para el IGS y factor de condición relativo. El ovario maduro presentó 7 fases de desarrollo de ovocitos, lo que sugiere que el desarrollo de los ovocitos es asincrónico. El arreglo de las células que se encontró en el testículo fue de tipo lobular, observándose de manera clara un conducto espermático bien definido de manera externa. La longitud de madurez fue de $25,4 \mathrm{~cm}$ para hembras y $24,5 \mathrm{~cm}$ para machos; debido a que estas longitudes son menores a las de captura promedio $(31,9$ y $29,7 \mathrm{~cm} \mathrm{LT})$ más del $50 \%$ de los organismos ya habrían tenido un evento reproductivo ( $99 \%$ de hembras y machos).

Palabras clave: Salmonete barbón, periodo reproductivo, longitud de maduración, proporción sexual, índice gonadosomático

\section{INTRODUCCIÓN}

Mulloidichthys dentatus (Gill, 1862) pertenece a la familia Mullidae que incluye 6 géneros y 55 especies distribuidas en mares templados y tropicales. La mayoría de las especies de esta familia habitan en el Indo-Pacífico occidental y solo dos especies son endémicas en el Pacífico oriental tropical (Robertson \& Allen 2015); algunos integrantes de la familia proporcionan información sobre procesos del ecosistema y se pueden usar como indicadores de integridad y el estado del hábitat natural (Uiblein 2007). La especie $M$. dentatus tiene amplia distribución geográfica en el Pacífico oriental tropical, desde Baja California Sur, México, hasta Perú, donde habita principalmente en arrecifes coralinos y rocosos, y también se encuentra en áreas adyacentes con fondos de arena y rocas (Robertson \& Allen 2015); se alimenta de organismos pequeños como crustáceos y poliquetos (Flores-Ortega et al. 2009). De M. dentatus solo se han podido localizar trabajos relacionados con listados faunísticos (Aguilar-Palomino et al. 1996, Lucano-Ramírez et al. 2001a), alimentación (Flores-Ortega et al. 2009) e información pesquera (Rojo-Vázquez et al. 2008). 
En otras partes del mundo se han desarrollado trabajos sobre la biología reproductiva de algunas especies de la familia Mullidae, como Pseudupeneus maculatus y Mulloidichthys martinicus en el Mar Caribe (Munro 1976); Mullus barbatus en el Mar Negro (Aydın \& Karadurmus 2013); Parupeneus multifasciatus en la bahía de NhaTrang, China (Pavlov et al. 2011), y varias especies del Mar Mediterráneo: Mullus surmuletus (Reñones et al. 1995, Murat-Kaya et al. 1999, Sieli et al. 2011, Arslan \& İşmen 2014); Upeneus moluccensis (İşmen 2005, 2006); Upeneus pori (Mehanna 2009, Kherraz et al. 2014) y Mullus barbatus (El-Drawany 2013, Arslan \& İşmen 2014). En México, son muy pocos los trabajos relacionados con la biología reproductiva de la familia Mullidae y solo se han localizado los realizados para la especie Pseudupeneus grandisquamis en el Golfo de Tehuantepec, Chiapas (Ramos-Santiago et al. 2006), en la Bahía Navidad, Jalisco (Lucano-Ramírez et al. 2006) y en el Golfo de California (Morales-Azpeitia et al. 2011).

Debido a la falta de información sobre la biología reproductiva de $M$. dentatus, se hace necesario conocer los principales aspectos reproductivos de la especie. Este tipo de investigaciones proporcionan información que pueden apoyar en la gestión del buen manejo de la pesquería y aportan conocimientos a otras investigaciones biológicas (Kjesbu 2009, Lowerre-Barbieri 2009). Con base en la información resumida anteriormente, el objetivo del presente trabajo fue investigar aspectos reproductivos de M. dentatus utilizando diferentes métodos para describir la temporada reproductiva que pueden ser importantes para su conservación y aprovechamiento sustentable.

\section{MATERIALES Y MÉTODOS}

El área de estudio es la Bahía de Navidad, Jalisco y Colima, ubicada en el litoral centro occidental mexicano del océano Pacífico (19¹0'30"-19¹2'50”N y 10442'45"$\left.104^{\circ} 41^{\prime} 30^{\prime \prime} \mathrm{W}\right)$. El lecho marino de esta región presenta fondos rocosos, fondos blandos y mixtos, destacan algunas estructuras de arrecifes de coral localizadas en zonas semiprotegidas. La plataforma continental, definida hasta la isobata de $200 \mathrm{~m}$, es angosta, con anchura que oscila entre 7 y 10 km (Rojo-Vázquez et al. 2001).

De enero 1998 a diciembre 2008, durante 5 días de cada mes, se muestrearon organismos capturados por pescadores ribereños utilizando redes de enmalle con diferentes tamaños de luz de malla $(7,62,8,89,10,16$ y $11,43 \mathrm{~cm}$ ). Los organismos capturados de Mulloidichthys dentatus fueron procesados, midiendo su longitud total (Lt; $\pm 0,1 \mathrm{~cm})$ y peso total $(\mathrm{Pt} ; \pm 0,1 \mathrm{~g})$; también se extrajeron las gónadas, las cuales se llevaron al laboratorio, donde se pesaron $(\mathrm{Pg} ; \pm 0,01 \mathrm{~g})$ y se les asignó el sexo, grado de madurez (Everson et al. 1989, Brown-Peterson et al.
2011), para luego preservarlas en formol neutro al 10\%. Posteriormente se incluyeron en parafina, se cortaron con un micrótomo rotatorio Arcano tipo Erma con grosor de 6 $\mu \mathrm{m}$ y se tiñeron con hematoxilina-eosina. La descripción de las fases de los ovocitos se basó en los trabajos realizados por Yamamoto \& Yamazaki (1961), Lucano-Ramírez et al. (2001b), Brown-Peterson et al. (2011) y Lowerre-Barbieri et al. (2011). Para evaluar el desarrollo reproductivo del testículo se consideraron los trabajos de Lucano-Ramírez et al. (2001c) y Brown-Peterson et al. (2011). Para el análisis mensual del diámetro de los ovocitos (DO), se analizaron 5 cortes histológicos de cada estadio macroscópico obtenido por mes ( $\sin$ considerar el año); de cada corte se midieron 10 ovocitos de las distintas fases observadas, esto mediante una cámara digital AxioCam ERc5s (Zeiss), acoplada a un microscopio AxioLabA1 (Zeiss); dichos diámetros resultaron del promedio entre el diámetro mayor y menor de cada ovocito; solo se midieron los ovocitos donde se observó el núcleo (West 1990, Lucano-Ramírez et al. 2016).

La temporada reproductiva se determinó mediante la combinación de tres métodos: en el primero se aplicó la escala morfocromática de maduración gonadal de Everson et al. (1989) y Brown-Peterson et al. (2011), considerando los altos porcentajes de organismos en estadios de mayor maduración por mes. El segundo método, fue el índice gonadosomático $\left(I G S=[P g / P t-P g]^{*} 100\right)$ el cual supone que los valores promedios máximos, indican la mayor madurez gonádica (Sánchez-Cárdenas et al. 2011). El tercer método consistió en conocer la variación mensual del diámetro promedio de los ovocitos, suponiendo que a un mayor diámetro le corresponde mayor grado de maduración ovárica.

Se obtuvo el factor de condición relativo $(\mathrm{FCr}=[\mathrm{Pt} /$ $\left.\left.L t^{2,92}\right]^{*} 100\right)$ como indicador del estado general de cada individuo, en donde 2,92 es el exponente de la relación longitud-peso $\left(P t=a * L t^{b}\right)$. Se analizó la variación mensual del IGS y FCr por sexo. También se calculó la proporción sexual por mes, por clase de longitud y para el total de organismos capturados. Se estimó la longitud a la cual el $50 \%$ de los individuos han alcanzado la madurez sexual $\left(L t_{50}\right)$, ajustando el porcentaje de organismos adultos (en desarrollo, capaz de reproducirse y desovados) de cada clase de longitud $\left(P_{L t}\right)$ al modelo logístico $\left(P_{L t}=\left[1 / 1+e^{(b L t+a)}\right]^{*} 100\right)$. El ajuste se realizó mediante un método de aproximación no lineal (algoritmo de Levenberg-Maquart).

Para el análisis estadístico de los datos, se utilizó la prueba Chi cuadrado $\left(\chi^{2}\right)$ de bondad de ajuste para dos categorías (Zar 2010) y determinar si la proporción de sexos difiere del valor teórico esperado $1 \mathrm{H}: 1 \mathrm{M}$. Se calcularon el valor promedio y error estándar $( \pm$ ee) por mes y se realizaron análisis de varianza (ANDEVA) para las variables IGS, FCr y DO. Cuando se encontró diferencia significativa 
en el ANDEVA, se utilizó la prueba de contrastes múltiples de Student-Newman-Keuls (SNK). Se realizaron análisis de correlación no paramétrica por rangos de Spearman $\left(\mathrm{r}_{\mathrm{s}}\right)$ entre el IGS y el FCr de cada sexo, y entre el IGS de hembras y DO. Todas las variables para su análisis se agruparon por mes sin considerar los años, y con esto se formó un año tipo. Para todos los análisis estadísticos se utilizó un nivel de significación $\alpha=0,05$. Las pruebas estadísticas se realizaron en el programa STATISTICA v7.1 (Statsoft 2006) ${ }^{1}$.

\section{Resultados}

\section{DiSTRIBUCIÓN DE LA LONGITUD TOTAL}

Fueron recolectados 1.590 organismos de Mulloidichthys dentatus. Estos presentaron longitud total de 16,5 a 40,0 cm y promedio de $30,9( \pm 0,09) \mathrm{cm}$. Las hembras presentaron valores de 20,2 a 40,0 cm y longitud promedio de 31,9 $( \pm 0,11) \mathrm{cm}$, en los machos se registraron longitud total entre 16,5 y $37,9 \mathrm{~cm}$ con promedio de $29,7( \pm 0,13) \mathrm{cm}$. La longitud promedio de las hembras fue significativamente mayor que la longitud de los machos $\left(\mathrm{F}_{1,1588}=181,9 ; P<\right.$ $0,001)$. En tres clases de longitud se concentró el $70 \%$ de hembras $(30,32,34 \mathrm{~cm})$ y el $65 \%$ de machos $(28,30,32$ cm) (Fig. 1).

\section{ESTADIOS DE MADUREZ GONÁDICA}

Los cuatro estadios de madurez (inmaduro, en desarrollo, capaz de reproducirse y desovado) se presentaron en ambos sexos en casi todos los meses. En las hembras el estadio mejor representado fue el capaz de reproducirse, principalmente en junio (69\%) y julio (72\%) y en los mismos meses se presentaron porcentajes bajos de organismos inmaduros (3\%) (Fig. 2a). Resultados similares se presentaron en los machos, en junio con el $60 \%$ de los organismos en estadio capaz de reproducirse y porcentaje bajo (4\%) de organismos inmaduros (Fig. 2b).

\section{ÍNDICE GONADOSOMÁTICO (IGS)}

Los valores del IGS en las hembras aumentaron de enero a julio, presentando los promedios máximos en junio y julio (2,87 y 2,83), con un descenso de agosto a octubre $(1,96$ a 1,93$)$, en noviembre se registró otro valor alto $(2,46)$ pero de menor magnitud que los que se presentaron en junio y julio. En los machos ocurrió algo semejante, el IGS disminuyó al inicio del año, y los valores promedios máximos se registraron en mayo, junio y julio $(2,15,2,68$ y 2,29 ), disminuyendo en la segunda mitad del año (agosto a diciembre). Se encontraron diferencias significativas entre los valores promedio mensuales en hembras $\left(\mathrm{F}_{11,899}=5,4 ; P\right.$ $<0,001)$, y machos $\left(\mathrm{F}_{11,691}=7,6 ; P<0,001\right)$. La prueba de contrastes múltiples identificó tres grupos en ambos sexos (a, b, c para hembras y 1, 2, 3 para machos), presentando traslapes en algunos meses. La variación mensual del IGS presentó un patrón similar en ambos sexos y la correlación entre los promedios mensuales del IGS de hembras y machos de $M$ dentatus fue significativa ( $\mathrm{r}_{\mathrm{s}}=0,594 ; P=0,042$; $\mathrm{n}=12$ ) (Fig. 3).

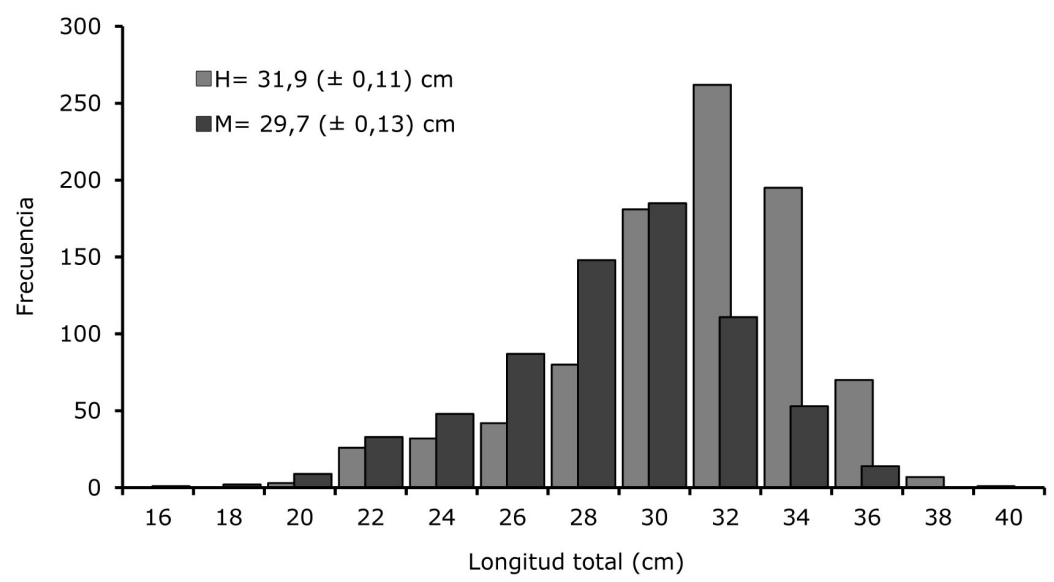

Figura 1. Distribución de las frecuencias de longitudes de hembras $(\mathrm{H})$ y machos $(\mathrm{M})$ de Mulloidichthys dentatus en el Pacífico tropical mexicano / Length frequency distribution of females (H) and males (M) of Mulloidichthys dentatus in the Mexican tropical Pacific

${ }^{1}$ StatSoft. 2006. STATISTICA (data analysis software system) version 7.1. <www.statsoft.com> 
a)

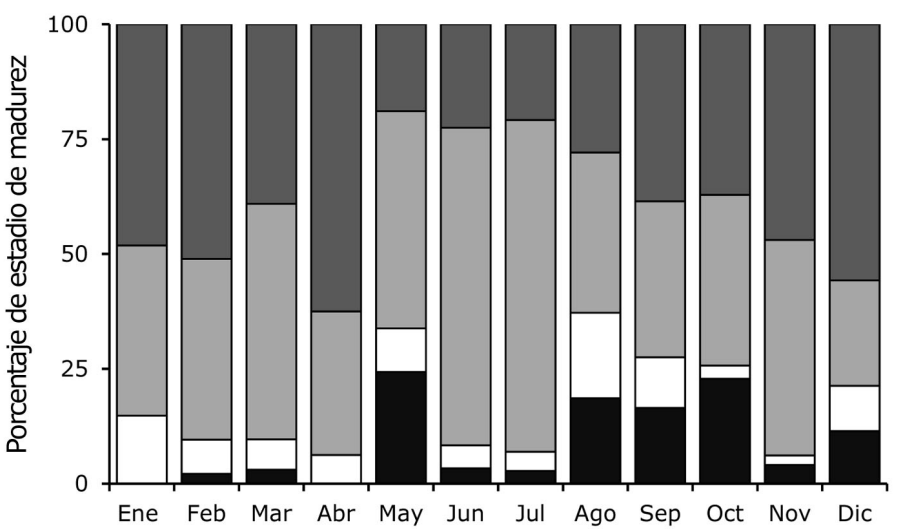

b)
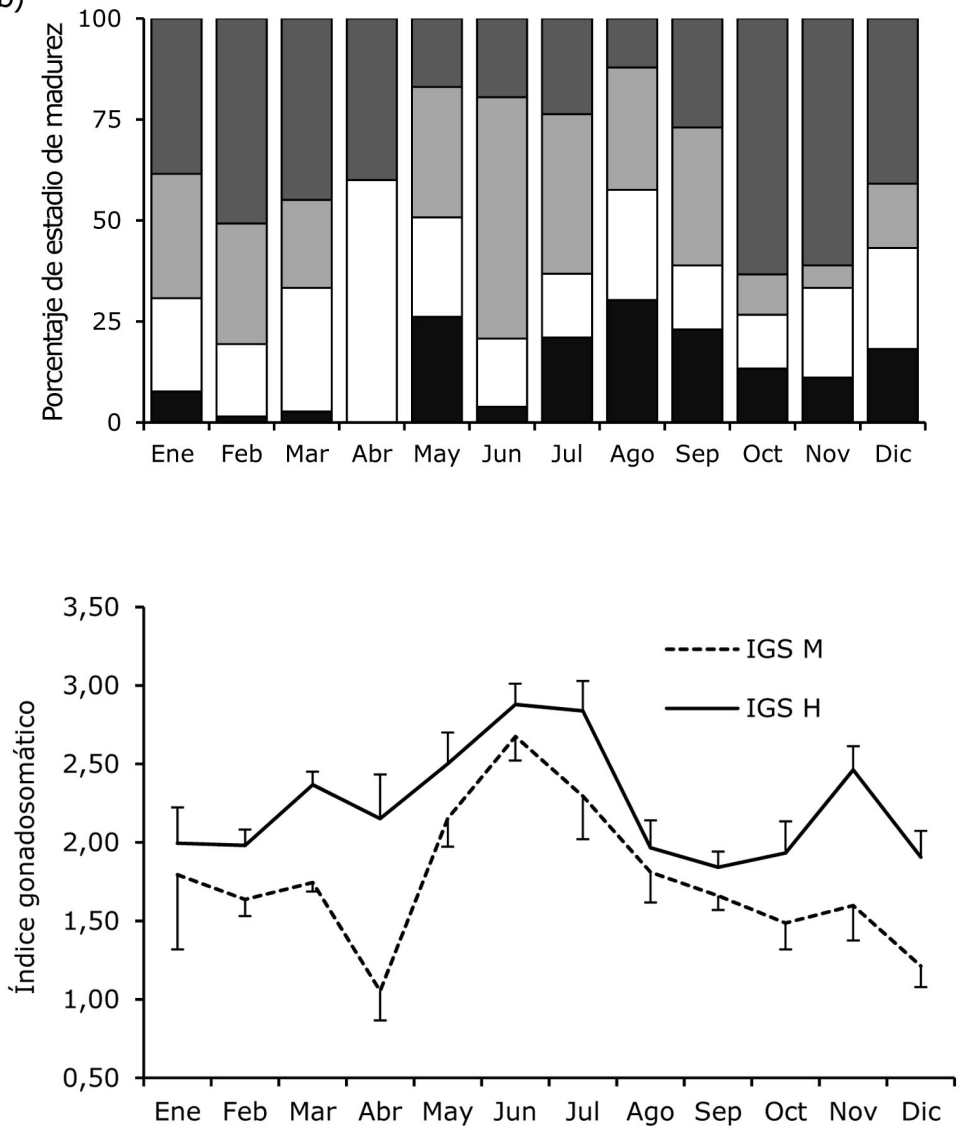

Figura 2. Porcentaje mensual de los estadios de madurez gonádica en hembras (a) y machos (b) de Mulloidichthys dentatus en el Pacífico tropical mexicano / Monthly percentage of gonadal maturity stages in females (a) and males (b) of Mulloidichthys dentatus in the Mexican tropical Pacific

Figura 3. Promedio mensual ( \pm error estándar) del índice gonadosomático (IGS) para hembras (H) y machos (M) de Mulloidichthys dentatus en el Pacífico tropical mexicano / Monthly mean (+ standard error) of gonadosomatic index for females $(\mathrm{H})$ and males $(\mathrm{M})$ of Mulloidichthys dentatus in the Mexican tropical Pacific

\section{FACTOR DE CONDICIÓN RELATIVO (FCR)}

El factor de condición relativo de hembras y machos presentó variación a lo largo del año, sin embargo se pudo observar un aumento en ambos sexos en mayo, posterior a este, disminuye (excepto en agosto y noviembre) hasta alcanzar su valor mínimo en septiembre en los machos $(0,84)$ y octubre en las hembras $(0,83)$ (Fig. 4). Se encontraron diferencias significativas entre los valores mensuales del FCr de hembras $\left(\mathrm{F}_{11,899}=8,2 ; P<0,001\right) \mathrm{y}$ de machos $\left(\mathrm{F}_{11,691}=\right.$ $6,8 ; P<0,001)$. La prueba de contrastes múltiples identificó 5 grupos (a, b, c, d, e) para hembras y 3 grupos $(1,2,3)$ para los machos. La prueba no paramétrica por rangos de Spearman indicó correlación entre los promedios mensuales del FCr de hembras y machos $\left(\mathrm{r}_{\mathrm{s}}=0,741 ; P=0,006 ; \mathrm{n}=12\right)$.

\section{VARIACIÓN CONJUNTA ENTRE EL IGS Y EL FCR}

En las hembras, el patrón de variación mensual del IGS y el FCr es similar, ya que presentaron correlación significativa $\left(\mathrm{r}_{\mathrm{s}}=0,657 ; P=0,024 ; \mathrm{n}=12\right)$. Lo anterior sugiere una sincronía temporal entre estas dos variables. Sin embargo en los machos la correlación entre el IGS y el FCr no fue significativa $\left(\mathrm{r}_{\mathrm{s}}=-0,482 ; P=0,112 ; \mathrm{n}=12\right)$, a pesar de que la variación en los promedios fue semejante en algunos meses. 


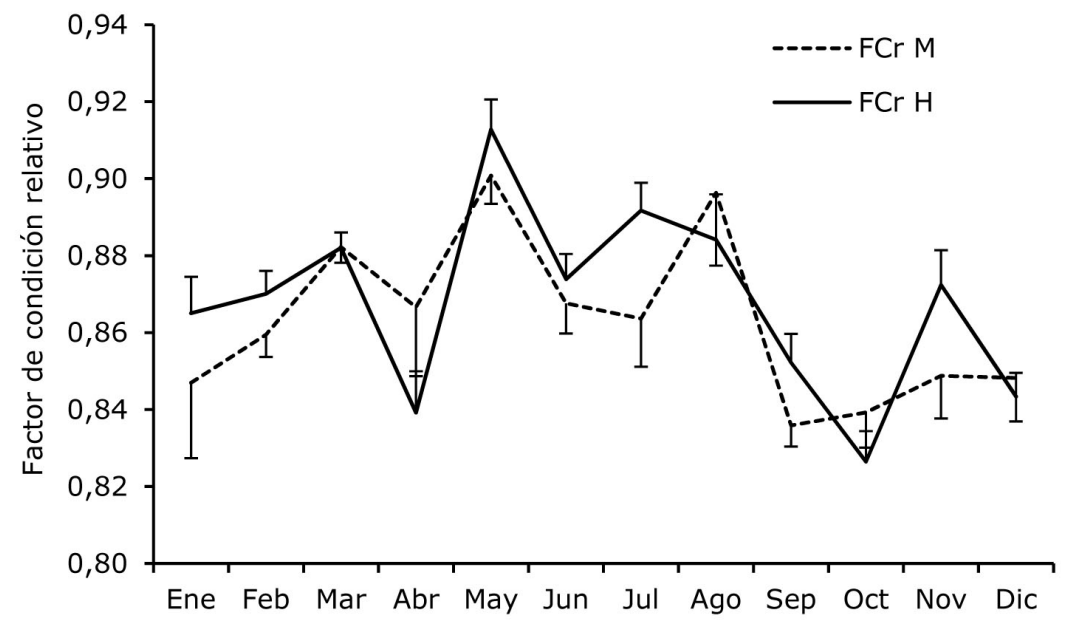

Figura 4. Promedio mensual ( \pm error estándar) del factor de condición relativo ( $\mathrm{FCr}$ ) de hembras (H) y machos (M) de Mulloidichthys dentatus en el Pacífico tropical mexicano / Monthly mean $( \pm$ standard error) of condition factor of females $(\mathrm{H})$ and males (M) of Mulloidichthys dentatus in the Mexican tropical Pacific

\section{DESCRIPCIÓN MICROSCÓPICA DE LAS GÓNADAS}

Los ovarios están cubiertos por una túnica ovárica, la cual presentó grosor de 9,12 a 399,58 $\mu \mathrm{m}$ y está formada por vasos sanguíneos y tejido muscular liso. Esta túnica se hace continua para formar crestas o lamelas al interior del ovario en numerosos pliegues y ramificaciones que ocupan la cavidad del ovario. En el interior del ovario se localizaron ovocitos en fase de crecimiento primario, esta fase fue la más pequeña $(53,76 \pm 0,44 \mu \mathrm{m})$ y abundante en todos los estadios de madurez (Fig. 5a). También se identificaron ovocitos con alveolos corticales $(125,58 \pm 0,80 \mu \mathrm{m})$ y ovocitos en distintas fases de la vitelogénesis: primaria $(209,14 \pm 0,77$ $\mu \mathrm{m})$, secundaria $(260,02 \pm 0,95 \mu \mathrm{m})$ y terciaria $(262,42 \pm$ $0,72 \mu \mathrm{m})$. Además, se identificaron ovocitos con vesícula germinal en migración $(232,43 \pm 5,38 \mu \mathrm{m})$. La presencia de diferentes fases de ovocitos en el ovario capaz de reproducirse de $M$. dentatus, nos permite deducir que los ovocitos se desarrollan de forma asincrónica (5b).

Los testículos están rodeados por la túnica albugínea, su interior está organizado por lóbulos, los cuales constan de diversos cistos, en donde ocurre todo el proceso de la espermatogénesis. Una vez formados los espermatozoides son liberados en el lumen de los lóbulos, posteriormente los espermatozoides se agrupan en grandes cantidades en un conducto espermático muy bien diferenciado cuando la gónada está en el estadio capaz de reproducirse. A este tipo de organización de las células en el interior del testículo se le clasifica de tipo lobular (Fig. 6).

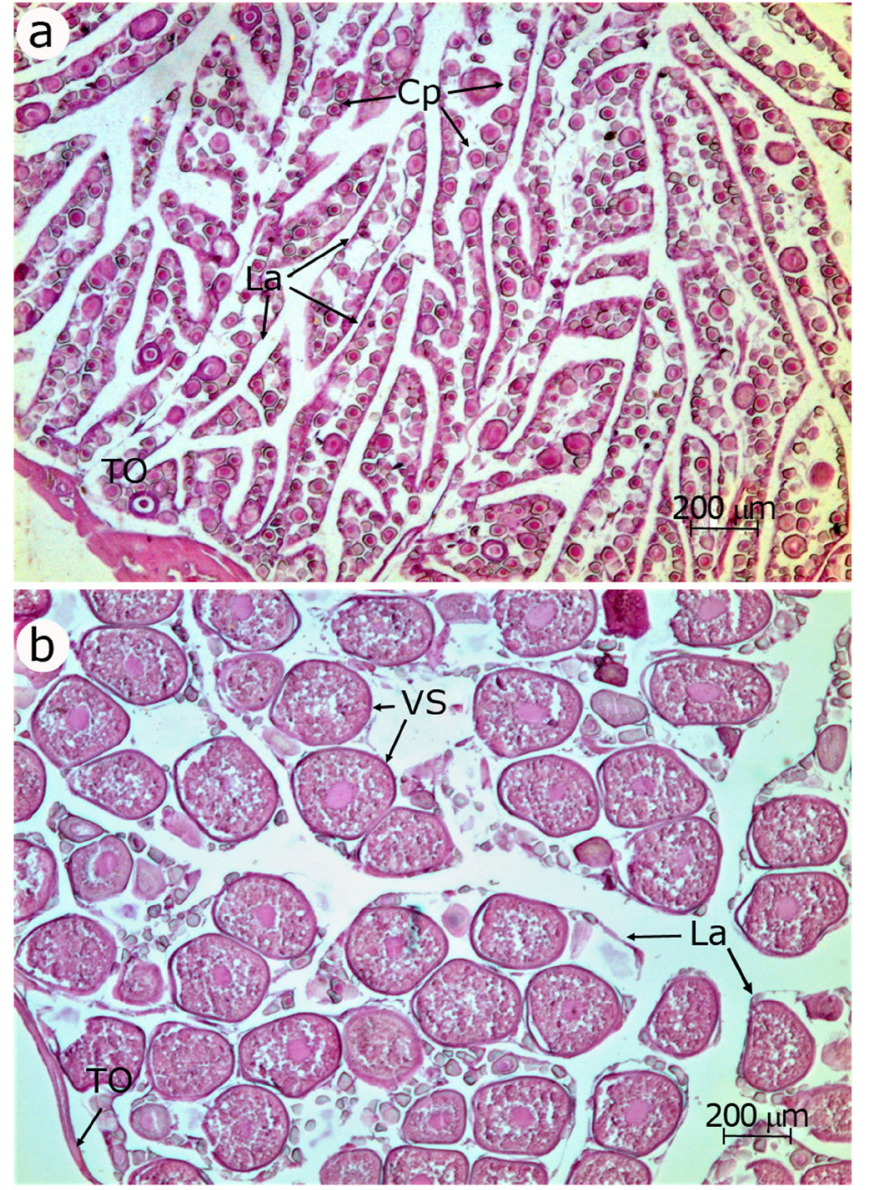

Figura 5. Ovario inmaduro (a) y maduro (b) de Mulloidichthys dentatus. $\mathrm{TO}=$ túnica ovárica, $\mathrm{La}=$ lamela, $\mathrm{Cp}=$ ovocitos en crecimiento primario, VS= vitelogénesis secundaria / Immature ovary (a) and mature ovary (b) of Mulloidichthys dentatus. TO $=$ tunica ovarica, $\mathrm{La}=$ lamella, $\mathrm{C} p=$ primary growth oocytes, VS= secondary vitellogenic 


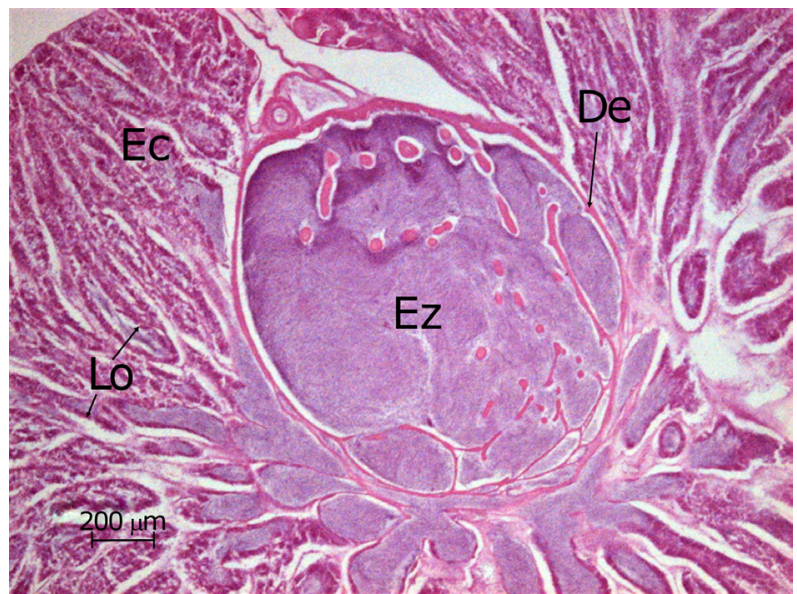

Figura 6. Testículo maduro de Mulloidichthys dentatus. De= Ducto espermático, Lo= lóbulos, Ec= espermatocitos, Ez= espermatozoides / Mature testes of Mulloidichthys dentatus. De= spermatic duct, Lo= lobules, $\mathrm{Ec}=$ spermatocytes, $\mathrm{Ez}=$ spermatozoa

\section{DiÁMETRO DE LOS OVOCITOS}

La variación del diámetro promedio de los ovocitos mostró diferencias significativas entre los meses $\left(\mathrm{F}_{11,3845}=14,13\right.$; $P<0,001)$. La prueba de contrastes múltiples identificó 6 grupos con traslapes en varios meses; el diámetro promedio menor se presentó en diciembre $(161,49 \pm 6,22 \mu \mathrm{m})$, mientras que el diámetro promedio mayor se presentó en junio $(210,45 \pm 3,53 \mu \mathrm{m})$, los meses restantes tienen valores promedio intermedios que difieren de estos dos. Se encontró correlación temporal $\left(\mathrm{r}_{\mathrm{s}}=0,923 ; P<0,001 ; \mathrm{n}=12\right)$, entre el diámetro promedio de ovocitos e IGS (Fig. 7).

\section{Proporción SeXual}

De los organismos analizados de $M$. dentatus, 899 (56,5\%) fueron hembras y $691(43,5 \%)$ machos, que corresponde a una proporción sexual de 1.3:1 H:M, la cual difiere significativamente $\left(\chi^{2}=27,21 ; P<0,001\right)$ de la esperada. En el análisis por meses, las hembras dominaron en número sobre los machos a excepción de septiembre, y en enero, febrero, marzo, junio, julio y noviembre la diferencia fue significativa (Tabla 1). Por clase de longitud total (LT), los machos dominaron sobre las hembras a los $<20,22,24,26$ y $28 \mathrm{~cm}$, en la LT de $30 \mathrm{~cm}$ la proporción fue 1:1 (H: M), sin embargo en los 32, 34 y >36 cm LT, dominaron las hembras. Se presentaron diferencias significativas en las longitudes totales de $<20,26,28,32,34$ y $>36 \mathrm{~cm}$ (Tabla 2).

\section{LONGITUD DE MADUREZ SEXUAL $\left(\boldsymbol{L}_{50}\right)$}

El 50\% de las hembras de $M$. dentatus alcanzaron la madurez sexual a los 25,4 cm LT, y los machos a los 24,5 cm LT (Fig. 8), la hembra más pequeña con gónadas maduras fue de 21,8 cm, y el macho de 21,3 cm LT. Por otra parte, los organismos más grandes con gónadas inmaduras fueron de $32,9 \mathrm{~cm}$ la hembra y $31,1 \mathrm{~cm}$ el macho. Más del $50 \%$ de los organismos capturados ya se habían reproducido al menos una vez debido a que la longitud promedio de captura fue mayor (31,9 cm LT las hembras y 29,7 cm LT los machos) a la $L_{50}$. Los machos alcanzaron la madurez sexual antes que las hembras.

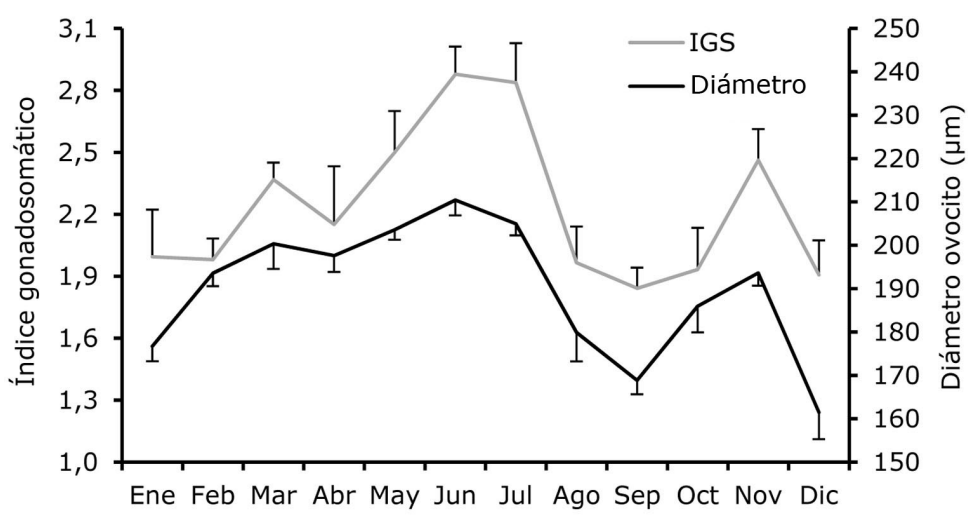

Figura 7. Promedio mensual ( \pm error estándar) del índice gonadosomático de hembras (IGS) y diámetro de los ovocitos de Mulloidichthys dentatus en el Pacífico tropical mexicano / Monthly mean ( + standard error) of gonadosomatic index and oocyte diameter of Mulloidichthys dentatus in the Mexican tropical Pacific 
Tabla 2. Proporción sexual por clase de longitud de Mulloidichthys dentatus en el Pacífico tropical mexicano / Sex ratio by class length of Mulloidichthys dentatus in the Mexican tropical Pacific

\begin{tabular}{ccccrc}
\hline $\begin{array}{c}\text { Longitud total } \\
(\mathrm{cm})\end{array}$ & Hembras & Machos & H:M & $\chi^{2}$ & $P$ \\
\hline$<20$ & 3 & 13 & $0,2: 1$ & 6,30 & $0,012^{*}$ \\
22 & 26 & 33 & $0,8: 1$ & 0,80 & 0,371 \\
24 & 32 & 48 & $0,7: 1$ & 3,20 & 0,073 \\
26 & 42 & 87 & $0,5: 1$ & 15,70 & $0,001^{*}$ \\
28 & 80 & 148 & $0,5: 1$ & 20,30 & $0,001^{*}$ \\
30 & 181 & 185 & $1,0: 1$ & 0,04 & 0,841 \\
32 & 262 & 111 & $2,4: 1$ & 61,10 & $0,001^{*}$ \\
34 & 195 & 53 & $3,7: 1$ & 81,30 & $0,001^{*}$ \\
$>36$ & 78 & 14 & $5,6: 1$ & 44,50 & $0,001^{*}$ \\
\hline
\end{tabular}

$\chi^{2}=$ valor obtenido de la prueba; $P=$ valor de probabilidad asociado;

*=indica diferencia estadística significativa

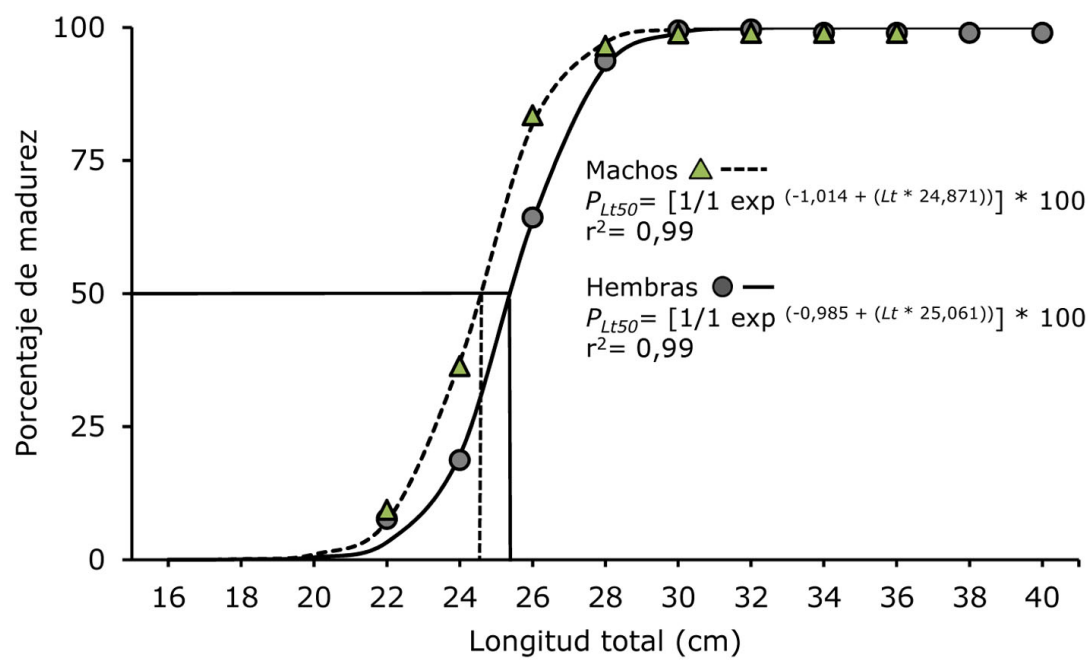

Figura 8. Porcentaje acumulado de individuos con gónadas maduras de hembras y machos de Mulloidichthys dentatus en el Pacífico tropical mexicano. Las líneas verticales indican el valor de $L t_{50}$ para cada sexo / Cumulative percentage of individuals with mature gonads in females and males of Mulloidichthys dentatus in the Mexican tropical Pacific. Vertical lines indicate the value of $L t_{50}$ for each sex

\section{Discusıón}

Las longitudes de los organismos de Mulloidichthys dentatus que se capturan en las pesquerías artesanales del Pacífico mexicano, solo han sido registradas para las costas de Jalisco y Colima. Dichas longitudes oscilan entre 12,1 a 40,0 cm de LT y provienen de organismos capturados con redes de enmalle, que son ampliamente utilizadas por pescadores ribereños (Espino-Barr et al. 2003, 2004; Flores-Ortega et al. 2009). No se encontró otro trabajo que hiciera referencia a las longitudes promedio de hembras y de machos de la especie. En este estudio se observó que las longitudes promedio de ambos sexos fueron diferentes. Las causas de este resultado podrían atribuirse al número de hembras en longitudes grandes y posiblemente a la segregación espacial. En este sentido, la proporción sexual puede cambiar de una población a otra y es un factor importante en la estructura de las poblaciones de peces (Nikolsky 1963).
Para $M$. dentatus, las hembras se presentaron en mayor número que los machos y al parecer, esta es una característica común que ocurre en otras especies que integran la familia Mullidae; en P. grandisquamis la proporción fue 1:0,3 H:M (Morales-Azpeitia et al. 2011), en M. surmuletus y U. pori se observaron más hembras que machos (El-Drawany 2013, Kherraz et al. 2014), en U. moluccensis la proporción favoreció a las hembras 1,1:1 (İşmen 2005) y 3:1 (MuratKaya et al.1999), lo mismo ocurrió con M. barbatus 2:1 H:M (Aydin \& Karadurmus 2013). En contraste, Ramos-Santiago et al. (2006) y Lucano-Ramírez et al. (2006) observaron proporciones $1: 1$ y $1: 1,15$ de hembras y machos para $P$. grandisquamis, sin diferencia significativa. 
Las escalas macroscópicas se basan en identificar cambios de apariencia y tamaño relativo en las gónadas, y son una herramienta utilizada al definir el ciclo reproductivo en peces (Karlou-Riga \& Economidis 1996). La presencia de gónadas maduras, se considera un indicador confiable de la temporada reproductiva (West 1990). Algunos trabajos con especies de la familia Mullidae, también utilizaron las características de las gónadas para delimitar el periodo reproductivo como en M. barbatus (Sieli et al. 2011), $P$. maculatus y M. martinicus (Munro 1976), P. grandisquamis (Lucano-Ramírez et al. 2006) y M. barbatus (Arslan \& İşmen 2014). En el presente trabajo, se observaron porcentajes altos de gónadas maduras en junio y julio en ambos sexos, además los estadios de madurez de las gónadas mostraron un patrón definido, con lo cual, se especifica parcialmente el periodo reproductivo.

El índice gonadosomático (IGS) es uno de los índices más utilizados como indicador del periodo reproductivo de muchas especies de peces de la familia Mullidae (Reñones et al. 1995, İşmen 2005, 2006; Lucano-Ramírez et al. 2006, Ramos-Santiago et al. 2006, Pavlov et al. 2011, Sieli et al. 2011, Aydin \& Karadurmus 2013, El-Drawany 2013, Kherraz et al. 2014, Arslan \& İşmen 2014). Los valores altos del IGS están asociados con la mayor actividad reproductiva y los valores bajos están relacionados con épocas de reposo gonadal (Htun-Han 1978). De acuerdo con los valores máximos del IGS de hembras y machos obtenidos en el presente estudio, $M$. dentatus presenta tres periodos reproductivos: uno a principios del año, en marzo; otro más amplio de mayo a julio y uno más a finales del año, en noviembre. Un patrón parecido fue reportado para otra especie de la familia Mullidae, P. grandisquamis, en el Pacifico central mexicano por Morales-Azpeitia et al. (2011). Estos autores encontraron que en el Golfo de California esta especie se reproduce principalmente en marzo y abril, con otro pico en septiembre. LucanoRamírez et al. (2006) mencionan que $P$. grandisquamis, se reproduce en mayo, noviembre y diciembre en la costa de Jalisco. Ramos-Santiago et al., (2006) registraron que $P$. grandisquamis en el Golfo de Tehuantepec se reproduce en marzo y octubre. Otras especies de la familia Mullidae presentan dos periodos reproductivos: M. martinicus, en el Mar Caribe (Munro 1976) y en el Mar Mediterráneo, M. surmuletus (Reñones et al. 1995, Kherraz et al. 2014), U. moluccensis (İşmen 2005), U. pori (İşmen 2006), M. barbatus (Sieli et al. 2011) y P. multifasciatus (Pavlov et al. 2011). En otros casos se reporta solamente un periodo reproductivo: U. moluccensis (Murat-Kaya et al. 1999), M. surmuletus (Mehanna 2009), U. pori (El-Drawany 2013) y M. barbatus (Arslan \& İşmen 2014) en el Mar Mediterráneo; y M. barbatus, en el Mar Negro (Aydin \& Karadurmus 2013). Las anteriores diferencias temporales que se registran en el periodo reproductivo, pueden deberse a que son especies diferentes y a las distintas características ambientales de las localidades geográficas. Otros estudios han observado que cada especie utiliza diferentes estrategias de reproducción adaptadas a múltiples estímulos del ambiente y los propios de cada especie durante sus ciclos de vida (Yamahira 2004).

Varios estudios han señalado que el FC constituye una medida de las reservas de energía relacionadas con condiciones ambientales, estado de madurez y alimentación e incluso en algunas especies los valores del FC se relacionan de forma inversa con los valores del IGS, lo cual se debe principalmente al gasto de energía utilizado en el proceso reproductivo o a la alternancia entre periodos de acumulación de reservas de energía y periodos de agotamiento debido a la reproducción; además también se ha observado un mayor gasto energético en la formación de la gónada en hembras que en machos (West 1990, King 1995, González \& Oyarzún 2002, González et al. 2006), sin embargo, en otras especies no se presenta esta relación inversa, y puede haber variación en la relación entre el FC e IGS. Este comportamiento se presentó en este estudio para $M$. dentatus; en las hembras, los promedios mensuales máximos presentaron una correlación positiva, lo cual indica una sincronía temporal entre FC e IGS. Aunque en los machos los valores promedios mensuales del FC y del IGS no se correlacionaron, algunos valores máximos de ambos índices coinciden, sobre todo en el periodo reproductivo (marzo y mayo).

En los ovarios capaces de reproducirse de $M$. dentatus se identificó el desarrollo asincrónico de los ovocitos. Al parecer no existen trabajos que describan el desarrollo de los ovocitos de la especie en estudio, sin embargo, otras especies de la familia Mullidae presentan el mismo tipo de desarrollo (P. grandisquamis, Lucano-Ramírez et al. 2006; P. multifasciatus, Pavlov et al. 2011; Emel'yanova et al. 2014). Este patrón de desarrollo que presentan los ovocitos, sugiere que los organismos desovan en varias ocasiones y pueden tener una temporada reproductiva prolongada (Nagahama 1983, Maack \& George 1999); por lo anterior, podemos sugerir que este tipo de desove es el que presenta M. dentatus.

Uribe et al. (2014) señalan que muchas especies de teleósteos presentan un tipo de testículo en donde el desarrollo de las espermatogonias no está restringido; en este tipo de testículo las espermatogonias se encuentran a todo lo largo de los lóbulos, en donde se lleva a cabo todo el proceso de la espermatogénesis. En este estudio $M$. dentatus mostró este mismo patrón de desarrollo y también fue reportado para $P$. grandisquamis por Lucano-Ramírez et al. (2006), de la misma familia. De igual forma, este mismo arreglo testicular se ha encontrado en otros teleósteos marinos como Diodon holocanthus (Lucano-Ramírez et al. 2011a), Scomberomorus sierra (Lucano-Ramírez et al. 2011b), Lutjanus argentiventris (Lucano-Ramírez et al. 
2014) y Carangoides victus (Lucano-Ramírez et al. 2016). Un aspecto interesante en $M$. dentatus es que desarrolla un conducto espermático en la parte externa de la gónada en donde los espermatozoides se concentran, en la mayoría de los peces el conducto espermático se encuentra en el interior del testículo.

La longitud promedio de madurez es una variable clave que puede ayudar a establecer medidas de regulación en las capturas (tallas mínimas de captura, luz de malla, grosor de hilo, entre otros), así como en el uso de diferentes artes de pesca (Saborido-Rey 2008). La longitud promedio de captura (31,9 cm LT las hembras y 29,7 cm LT los machos) representó el 99\% de hembras y el 99\% de machos sexualmente maduros, la longitud promedio de captura fue mayor a la $L_{50}$ para ambos sexos; este mismo patrón en donde más del $50 \%$ de los organismos ya se han reproducido antes de ser capturados se ha reportado para otras especies de la familia Mullidae, como P. grandisquamis en donde el $61 \%$ de hembras y el $60 \%$ de machos eran sexualmente maduros (Lucano-Ramírez et al. 2006), en U. moluccensis el 50\% en ambos sexos (İşmen 2005), mientras que el 52\% de los organismos capturados de hembras y machos de $U$. pori (İşmen 2006) y en otro trabajo con la misma especie el 53 y 52\%, respectivamente (El-Drawany 2013).

Al parecer los machos de $M$. dentatus maduran sexualmente primero que las hembras, esta es una característica que se ha observado en otras especies de esta familia, por ejemplo en M. surmuletus (Reñones et al. 1995), en $P$. grandisquamis (Lucano-Ramírez et al. 2006), en $U$. pori (El-Drawany 2013) y en M. barbatus (Arslan \& İşmen 2014). El hecho de que los machos alcancen la madurez sexual antes que las hembras podría ser una estrategia reproductiva, para asegurar la presencia de reproductores machos al momento de los primeros desoves de las hembras (Blancas-Arroyo 2008) y como en algunas especies los machos después de alcanzar una longitud suficiente dirigen su energía más a la reproducción y menos al crecimiento corporal (Hernández-Olalde 2008).

Debido al largo tiempo en que se llevó a cabo este estudio, los datos sobre los aspectos reproductivos que se encontraron para la especie $M$. dentatus se pueden tomar como referencia para futuras medidas de explotación.

\section{Agradecimientos}

A Manuel Díaz, Daniel y Gerado Kosonoy, por la ayuda en actividad de pesca. A las cooperativas pesqueras "Rivera de Melaque" y "Punta Farallón". A la Universidad de Guadalajara por el financiamiento y a estudiantes del DEDSZC, que colaboraron en la obtención de datos biológicos y en parte del trabajo de laboratorio.

\section{LITERATURA CITADA}

Aguilar-Palomino B, J Mariscal-Romero, LE RodríguezIbarra \& G González-Sansón. 1996. Ictiofauna demersal de fondos blandos de la plataforma continental de Jalisco y Colima, México, en la primavera de 1995. Ciencias Marinas 22: 469-481.

Arslan M \& A İşmen. 2014. Age, growth, reproduction and feeding of Mullus barbatus in Saros Bay (North Aegean Sea). Journal Black Sea/Mediterranean Environment 20: 184-199.

Aydın M \& U Karadurmuş. 2013. An investigation on age, growth and biological characteristics of red mullet (Mullus barbatus ponticus, Essipov, 1927) in the Eastern Black Sea. Iranian Journal of Fisheries Sciences 12: 277-288.

Blancas-Arroyo GA. 2008. Desarrollo ovárico y su relación con los niveles hormonales circulantes de $17 \beta$-estradiol y $17 \alpha$-hidroxiprogesterona durante el primer ciclo reproductivo en hembras de pez blanco Chirostoma humboldtianum (Valenciennes 1835). Veterinaria México 39: 67-80.

Brown-Peterson NJ, DM Wyanski, F Saborido-Rey, BJ Macewicz \& SK Lowerre-Barbieri. 2011. A standardized terminology for describing reproductive development in fishes. Marine and Coastal Fisheries: Dynamics, Management, and Ecosystem Science 3: 52-70.

El-Drawany MA. 2013. Some biological aspects of the Por's goatfish, (Family: Mullidae) from Tripoli cost of Libya. Egyptian Journal of Aquatic Research 39: 261-266.

Emel'yanova NG, DA Pavlov \& ED Pavlov. 2014. Anomalies in ovarian condition of many bar goatfish Parupeneus multifasciatus (Mullidae) from the coastal zone of south central Vietnam. Journal of Ichthyology 54: 76-84.

Espino-Barr E, M Cruz-Romero \& A García-Boa. 2003. Peces marinos con valor comercial de la costa de Colima, México, 106 pp. CONABIO, INAPESCA, CRIP, Manzanillo.

Espino-Barr E, EG Cabral-Solís, A García-Boa \& M PuenteGómez. 2004. Especies marinas con valor comercial de la costa de Jalisco, México, 145 pp. INP, SAGARPA, Manzanillo.

Everson AR, HA Williams \& BM Ito. 1989. Maturation and reproduction in two Hawaiian eteline snappers, uku, Aprion virescens, and onaga, Etelis coruscans. Fishery Bulletin 87: 877-888.

Flores-Ortega JR, E Godínez-Domínguez, JA Rojo-Vázquez, A Corgos, VH Galván-Piña \& G González-Sansón. 2009. Interacciones tróficas de las seis especies de peces más abundantes en la pesquería artesanal en dos bahías del Pacífico central mexicano. Revista de Biología Tropical 58: 383-397.

González P \& C Oyarzún. 2002. Variabilidad de índices biológicos en Pinguipes chilensis Valenciennes 1833 (Perciformes, Pinguipedidae): ¿Están realmente correlacionados? Gayana 66: 249-253.

González C, E Román \& X Paz. 2006. Condition and feeding of American plaice (Hippoglossoides platessoides) in the north Atlantic with emphasis on the flemish cap. Journal of Northwest Atlantic Fishery Science 37: 87-102. 
Hernández-Olalde L. 2008. Ecología reproductiva de peces de arrecifes rocosos en el suroeste del Golfo de California. Tesis Doctoral, CICIMAR-IPN, La Paz, 184 pp.

Htun-Han M. 1978. The reproductive biology of the dab Limanda limanda (L.) in the North Sea: Seasonal changes in the ovary. Journal of Fish Biology 13: 315-359.

İşmen A. 2005. Growth and reproduction of the goldband goatfish, Upeneus moluccensis (Bleeker, 1855), in Iskenderun bay, the Eastern Mediterranean. Turkish Journal of Zoology 29: 301-309.

İşmen A. 2006. Growth and reproduction of por's goatfish (Upeneus pori Ben-Tuvia \& Golani, 1989) in Iskenderun bay, the Eastern Mediterranean. Turkish Journal of Zoology 30: 91-98.

Karlou-Riga C \& PS Economidis. 1996. Ovarian atretic rates and sexual maturity of horse mackerel, Trachurus trachurus (L.) in the Saronikos Gulf (Greece). Fishery Bulletin 94: 66-76.

Kherraz A, A Kherraz, S Benghali, S Mouffok \& Z Boutiba. 2014. Reproductive biology and growth of red mullet, Mullus surmuletus (Linne, 1758) in western Algeria coasts. Journal Academica 4: 121-129.

King MG. 1995. Fisheries biology, assessment and management, 400 pp. Oxford Fishing News Books, Oxford.

Kjesbu OS. 2009. Applied fish reproductive biology: contribution of individual reproductive potential to recruitment and fisheries management. In: Jakobsen T, MJ Fogarty, BA Megrey \& E Moksness. (eds). Fish reproductive biology: implications for assessment and management, pp. 293-332. Wiley-Blackwell Scientific Publications, Oxford.

Lowerre-Barbieri SK. 2009. Reproduction in relation to conservation and exploitation of marine fishes. In: Jamieson BGM (eds). Reproductive biology and phylogeny of fishes (agnathans and bony fishes) 8: 371-394. Science Publishers, Enfield.

Lowerre-Barbieri SK, NJ Brown-Peterson, H Murua, J Tomkiewicz, DM Wyanski \& F Saborido-Rey. 2011. Emerging issues and methodological advances in fisheries reproductive biology. Marine and Coastal Fisheries: Dynamics, Management, and Ecosystem Science 3: 32-51.

Lucano-Ramírez G, S Ruiz-Ramírez, B Aguilar-Palomino \& JA Rojo-Vázquez. 2001a. Listado de las especies de peces de la región costera de Jalisco y Colima, México. Ciencia y Mar 5: 13-20.

Lucano-Ramírez G, M Villagrán-Santa Cruz, S RuizRamírez \& MT López-Trujillo. 2001b. Histology of the oocytes of Lutjanus peru (Nichols and Murphy 1922) (Pisces: Lutjanidae). Ciencias Marinas 27: 335-349.

Lucano-Ramírez G, M Villagrán-Santa Cruz, S RuizRamírez. 2001c. Cambios estructurales en las gónadas de Lutjanus peru (Pisces: Lutjanidae), en la costa sur de Jalisco, México. Boletín del Centro de Investigaciones Biológicas 35: 295-316.

Lucano-Ramírez G, S Ruiz-Ramírez \& JA Rojo-Vázquez. 2006. Composición por tallas y ciclo reproductivo de Pseudupeneus grandisquamis (Pisces: Mullidae) en el Pacífico central mexicano. Revista de Biología Tropical 54: 195-207.
Lucano-Ramírez G, E Peña-Pérez, S Ruiz-Ramírez, JA Rojo-Vázquez \& G González-Sansón. 2011a. Aspectos reproductivos del pez erizo, Diodon holocanthus (Pisces: Diodontidae) en la plataforma continental del Pacífico central mexicano. Revista de Biología Tropical 59: 217-232.

Lucano-Ramírez G, S Ruiz-Ramírez, FI Palomera-Sánchez \& G González-Sansón. 2011b. Biología reproductiva de la sierra Scomberomorus sierra (Pisces, Scombridae) en el Pacífico central mexicano. Ciencias Marinas 37: 249-260.

Lucano-Ramírez G, S Ruiz-Ramírez, G González-Sansón \& BP Ceballos-Vázquez. 2014. Biología reproductiva del pargo alazán, Lutjanus argentiventris (Pisces, Lutjanidae), en el Pacífico central mexicano. Ciencias Marinas 40: 33-44.

Lucano-Ramírez G, EG Rivera-Ríos, S Ruiz-Ramírez, G González-Sansón \& A Perez-Toledo. 2016. Reproducción de Carangoides vinctus (Perciformes: Carangidae) en el Pacífico central mexicano. Latin American Journal of Aquatic Research 44: 610-622.

Maack G \& MR George. 1999. Contributions to the reproductive biology of Encrasicholina punctifer Fowler 1938 (Engraulidae) from West Sumatra, Indonesia. Fisheries Research 44: 113-120.

Mehanna SF. 2009. Growth, mortality and spawning stock biomass of the striped red mullet Mullus surmuletus, in the Egyptian Mediterranean waters. Mediterranean Marine Science 10: 5-17.

Morales-Azpeitia R, J López-Martínez, J Rodríguez-Romero \& JT Ponce-Palafox. 2011. Distribución, abundancia y patrón reproductivo de Pseudupeneus grandisquamis (Gill, 1863) (Perciformes: Mullidae) y Urobatis halleri Cooper, 1863 (Rajiformes: Urolophidae) en el Golfo de California. Investigación y Ciencia 52: 3-14.

Munro JL. 1976. Aspects of the biology and ecology of Caribbean reef fishes: Mullidae (goat-fishes). Journal of Fish Biology 9: 79-97.

Murat-Kaya M, HA Benli, T Katagan \& O Ozaydin. 1999. Age, growth, sex ratio, spawning season and food, of golden banded goatfish, Upeneus molucensis (Bleeker, 1855) from the Mediterranean and south Aegean Sea coasts of Turkey. Fisheries Research 41: 317-328.

Nagahama Y. 1983. The functional morphology of teleosts gonads. In: Hoar WS, DJ Randall \& EM Donaldson (eds). Fish physiology 9, Reproduction: 223-276. Academic Press, New York.

Nikolsky GV. 1963. The ecology of fish, 329 pp. Academic Press, New York.

Pavlov DA, NG Emel'yanova, TBT Luong \& H VoThi. 2011. Reproduction and initial development of many bar goatfish Parupeneus multifasciatus (Mullidae). Journal of Ichthyology 51: 628-641.

Ramos-Santiago E, JM Ramírez-Gutiérrez, R MendozaRodríguez \& M Tapia-García. 2006. Reproducción, distribución y abundancia del pez Pseudupeneus grandisquamis (Perciformes: Mullidae), en el Golfo de Tehuantepec, México. Revista de Biología Tropical 54: 1103-1112. 
Reñones O, E Massuti \& OB Morales. 1995. Life history of the red mullet Mullus surmuletus from the bottom-trawl fishery off the island of Majorca (north-west Mediterranean). Marine Biology 123: 411-419.

Robertson DR \& GR Allen. 2015. Peces costeros del Pacífico Oriental tropical: sistema de información en línea. Versión 2.0. Instituto Smithsonian de Investigaciones Tropicales, Balboa. <https://www.discoverlife.org/shorefish/s_index. html>

Rojo-Vázquez JA, B Aguilar-Palomino, VH Galván-Piña, E Godínez-Domínguez, S Hernández-Vázquez, S RuizRamírez \& G Lucano-Ramírez. 2001. Ictiofauna de la pesquería ribereña en Bahía Navidad, Jalisco, México asociada al evento El Niño 1997-1998. Revista de Biología Tropical 49: 841-854.

Rojo-Vázquez JA, C Quiñones-Velázquez, HA EchavarriaHeras, G Lucano-Ramírez, E Godínez-Domínguez, S Ruiz-Ramírez, VH Galván-Piña \& O Sosa-Nishizaki. 2008. The fish species composition and variation of catch from the small-scale gillnet fishery before, during and after the 1997-1998 ENSO event, central Mexican Pacific. Revista de Biología Tropical 56: 133-152.

Saborido-Rey F. 2008. Ecología de la reproducción y potencial reproductivo en las poblaciones de peces marinos, $71 \mathrm{pp}$. Curso de Doctorado, Instituto de Investigaciones Marinas (CSIC), Universidad de Vigo, Vigo.
Sánchez-Cárdenas R, M Arellano-Martínez, MC ValdezPineda, RE Morán-Angulo \& BP Ceballos-Vázquez. 2011. Reproductive cycle and sexual maturity of Sphoeroides annulatus (Jenyns, 1842) (Tetraodontiformes, Tetraodontidae) from the coast of Mazatlan, Sinaloa, Mexico. Journal of Applied Ichthyology 27: 1190-1196.

Sieli G, C Badalucco, G Di Stefano, P Rizzo, G D'Anna \& F Fiorentino. 2011. Biology of red mullet, Mullus barbatus (L. 1758), in the Gulf of Castellammare (NW Sicily, Mediterranean Sea) subject to a trawling ban. Journal of Applied Ichthyology 27: 1218-1225.

Uiblein F. 2007. Goatfishes (Mullidae) as indicators in tropical and temperate coastal habitat monitoring and management. Marine Biology Research 3(5): 275-288.

Uribe MC, HJ Grier \& V Mejía-Roa. 2014. Comparative testicular structure and spermatogenesis in bony fishes. Spermatogenesis 4(3): 1-13.

West G. 1990. Methods of assessing ovarian development in fishes: A review. Australian Journal of Marine and Freshwater Research 41(2): 199-222.

Yamahira K. 2004. How do multiple environmental cycles in combination determine reproductive timing in marine organisms? A model and test. Functional Ecology 18: 4-15.

Yamamoto K \& F Yamazaki. 1961. Rhythm of development in the oocyte of the gold-fish, Carassius auratus. Bulletin of the Faculty Fisheries, Hokkaido University 12: 93-114.

Zar JH. 2010. Biostatistical analysis, 960 pp. Prentice Hall, Englewood Cliffs. 